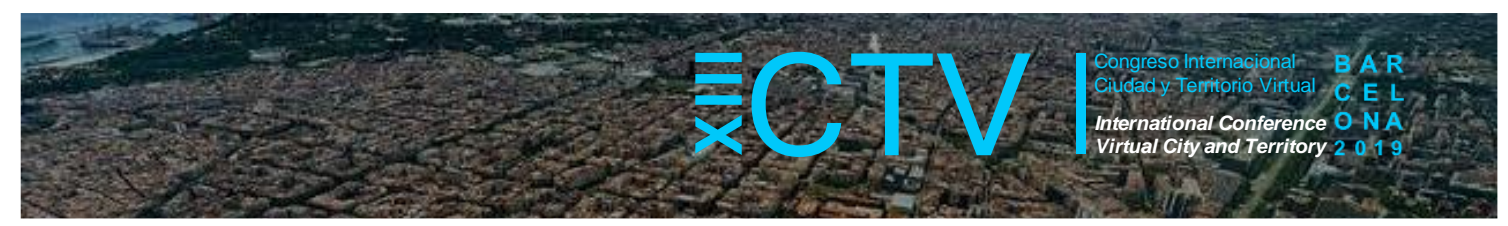

\title{
COOPERATIVAS VECINALES PARA EL ENVEJECIMIENTO ACTIVO EN BARRIADAS OBSOLETAS. IMPLICACIONES EN LA MEJORA DEL MERCADO DE ALQUILER SOCIAL
}

\author{
Rosa-Jiménez, Carlos ${ }^{1 *}$ y Nebot, Nuria ${ }^{2}$ \\ Remisión inicial: 2019-06-16; Remisión definitiva: 2019-10-20; Publicación: 2019-12-21
}

Citación: Rosa-Jiménez, C. y Nebot, N. (2019). Cooperativas vecinales para el envejecimiento activo en barriadas obsoletas. Implicaciones en la mejora del mercado de alquiler. En XIII CTV 2019 Proceedings: XIII International Conference on Virtual City and Territory: "Challenges and paradigms of the contemporary city": UPC, Barcelona, October 2-4,2019. Barcelona:CPSV, 2019, p. 8649. E-ISSN2604-6512. DOI http://dx.doi.org/10.5821/ctv.8649

\section{Resumen}

En el año 2050 la población mayor de 65 años llegará a 2.000 millones en todo el mundo. En España -de forma similar al resto de países del área meridional europea-, el proceso de envejecimiento de la población se inició más tarde, pero con una intensidad superior que en el resto de países de su entorno. Según la Encuesta Continua de Hogares de 2017, aproximadamente el $10 \%$ de los hogares españoles está habitado por personas mayores que viven en soledad. Las personas mayores que han vivido mucho tiempo en una comunidad prefieren envejecer en ellas y no desplazarse mientras dure sus redes sociales tradicionales y comunitarias. Además de la soledad, el envejecimiento en el lugar cuenta con otros dos grandes retos: por un lado, la mayor parte las personas mayores cuentan con unas viviendas sobredimensionadas que ya no se ajustan a sus necesidades habitacionales reales, razón por la que suelen venderlas y adquirir una vivienda más reducida cerca de sus hijos o hijas; y por otro, la incertidumbre de las pensiones ponen en riesgo la capitalización necesaria para llevar a cabo una reforma o contar con asistencia profesional de apoyo. Además de este problema, en las grandes ciudades turísticas españolas se consolida la falta de alquileres por efecto de las nuevas economías colaborativas. El alquiler de larga estancia (destinado principalmente a residentes) sufre actualmente una importante competencia por el alquiler de corta estancia (destinado principalmente a visitantes y turistas). La respuesta de la Administración a este fenómeno es el desarrollo de un cuerpo normativo que intenta regularlo o prohibirlo. Sin embargo, las Administraciones con competencia en materia de vivienda no son capaces de ofrecer nuevos modelos que contrarresten este imparable proceso. Como alternativa, este artículo demuestra que el modelo de una cooperativa vecinal de personas mayores en una barriada es económicamente viable, ya que resuelve el problema de la soledad mediante el agrupamiento de cooperativistas en viviendas adaptadas, liberando el resto de viviendas, que pasarían a formar parte de la oferta de viviendas de alquiler de larga temporada, gestionada por la propia cooperativa. Mediante un estudio económico de ingresos y gastos, se analiza la viabilidad económica de su implantación en dos barriadas de estudio (Sixto y Cortijo Vallejo), en un sector de barriadas sociales construidas entre 1960 y 70 en Málaga (España), con un importante nivel de obsolescencia física y funcional. Los resultados indican que la cooperativa vecinal de personas mayores permite disminuir los problemas de soledad de las personas mayores y aumentar la oferta de vivienda de alquiler. Además de la soledad, este modelo da respuesta a la incertidumbre económica de las pensiones y las necesidades especiales de habitabilidad. De esta forma, las personas mayores pueden adaptar aquellas viviendas que están mejor localizadas para sus necesidades, mientras que el alquiler de las viviendas restantes permite la capitalización de los recursos económicos de los socios de la cooperativa. Frente a otros modelos, como el de la co-vivienda o la regeneración urbana con subvención pública, la cooperativa vecinal de personas mayores fomenta la economía social, y el envejecimiento en el lugar. Todo ello desde la autosuficiencia económica, lo que permite su aplicación en momentos de crisis económica. Pero, fundamentalmente ofrece una herramienta que pueden utilizar las administraciones para incrementar el mercado de vivienda de alquiler, especialmente para aquellas familias con peligro de exclusión social, ya que favorece el alquiler a precios tasados. La implementación de este modelo podría resolver hasta el $50 \%$ de las necesidades de alquiler en la ciudad de estudio. Finalmente, el artículo propone futuras líneas de investigación, como el análisis sociológico del modelo y la necesidad de ampliar la rehabilitación de las viviendas a todos los socios cooperativistas.

\footnotetext{
1 Instituto Interuniversitario UMA-UPC Habitat, Turismo Territorio. Universidad de Málaga, https://orcid.org/0000-00016356-8734; 2 Instituto Interuniversitario UMA-UPC Habitat, Turismo Territorio. Universidad de Málaga,
} https://orcid.org/0000-0001-6187-0972. * Correo de contacto: cjrosa@uma.es 


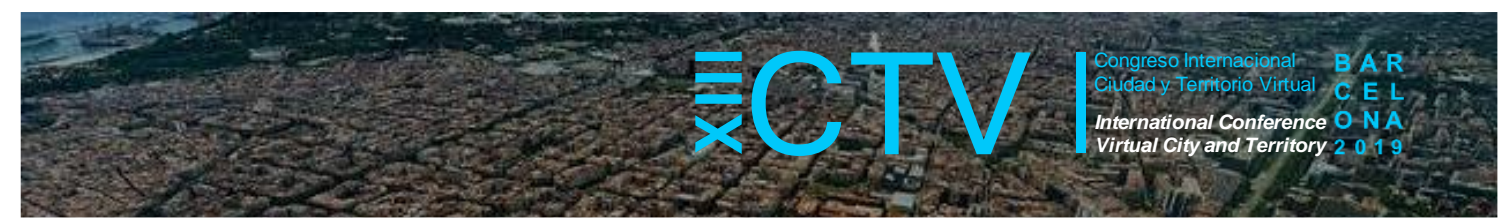

\section{Abstract}

In 2050 the population over 65 will reach 2,000 million worldwide. In Spain -like the rest of the countries in the southern European area-, the aging process of the population started later but with a higher intensity than in the rest of the surrounding countries. According to the 2017 Continuous Household Survey, elderly people who live alone inhabit approximately $10 \%$ of Spanish households. Older people who have lived in a community for a long time prefer to grow old in them and not move around while their traditional and community social networks last. In addition to loneliness, aging in the place has two other great challenges: on the one hand, most older people have oversized homes that no longer fit their real housing needs, which is why they tend to sell them and acquire a smaller house near your sons or daughters; and on the other, the uncertainty of pensions puts at risk the capitalization necessary to carry out a reform, to have professional support assistance. In addition to this problem, in the large Spanish tourist cities, the lack of rents due to the effect of the new collaborative economies is consolidated. Long-term rental (intended primarily for residents) currently suffers from significant competition for short-term rental (primarily intended for visitors and tourists). The administration's response to this phenomenon is the development of a regulatory body that attempts to regulate or prohibit it. However, administrations with jurisdiction over housing are not able to offer new models that counteract this unstoppable process. As an alternative, this article shows that the model of a neighborhood cooperative for older people in a neighborhood is an economically viable model that solves the problem of loneliness by grouping cooperative members in adapted housing, freeing the rest of the homes that would become part of the offer of long-term rental housing, managed by the cooperative itself. Through an economic study of income and expenses, the economic viability of its implementation in two study neighborhoods (Sixto and Cortijo Vallejo) is analyzed, in a sector of social neighborhoods built between 1960 and 70 in Malaga (Spain), with an important level of physical and functional obsolescence. The results indicate that the neighborhood cooperative for older people reduces the loneliness problems of older people and increases the supply of rental housing. In addition to loneliness, this model responds to the economic uncertainty of pensions and special needs for habitability. In this way, older people can adapt those homes that are best located for their needs, while renting the remaining homes allows capitalization of the economic resources of older people. Compared to other models, such as co-housing or urban regeneration with a public subsidy, the neighborhood cooperative for older people fosters the social economy and aging in place. Its self-sufficiency allows its application in times of economic crisis. But, fundamentally, it offers a tool that administrations can use to increase the rental housing market, especially for those families at risk of social exclusion, since it favors rental at appraised prices. The implementation of this model could solve up to $50 \%$ of the rental needs in the study city. Finally, the article proposes future lines of research, such as the sociological analysis of the model and the need to extend the rehabilitation of housing to all cooperative members.

Palabras Clave: envejecimiento; soledad; vivienda de alquiler; apoyo mutuo

Key words: ageing; loneliness; rental housing; mutual support

\section{Introducción. El problema del envejecimiento de la población y la falta de alquileres.}

\subsection{El envejecimiento de la población y el incremento de la soledad de las personas mayores}

En el año 2050 la población mayor de 65 años llegará a 2.000 millones en todo el mundo. En España y en el resto de países del área meridional europea, el proceso de envejecimiento de la población se inició más tarde pero con una intensidad superior que en el resto de países de su entorno (Fernández Muñoz, 2015, pág. 25). En 2013, ya se había duplicado la población de personas mayores de 1981. Para el año 2066 se estima que exista más de 14 millones de personas mayores, el $34,6 \%$ de un total de 41.068 .643 habitantes (Abellán García, Ayala García, \& Pujol Rodríguez, 2017, pág. 3).

Hui, Wong, Chung, \& Lau, (2014) han demostrado que las personas mayores que han vivido mucho tiempo en una comunidad prefieren envejecer en ellas y no desplazarse mientras dure 


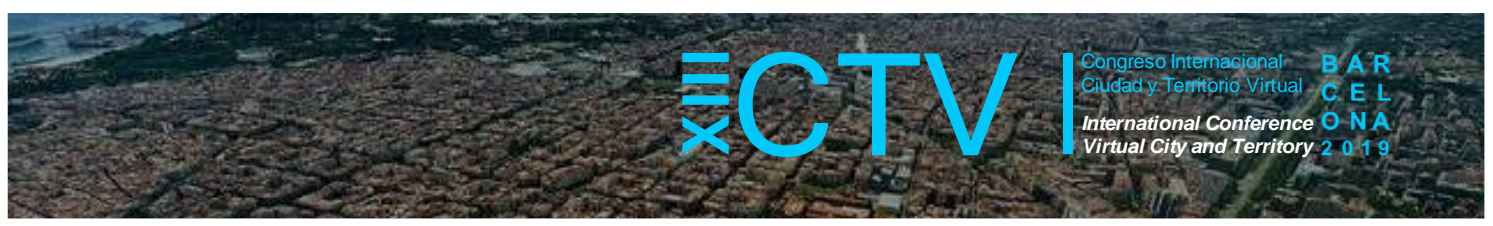

sus redes sociales tradicionales y comunitarias. De acuerdo con Wiesel (2012), vivir en el propio lugar tiene dos grandes ventajas: (1) es más económico que desplazarse a otro tipo de centros especializados (como las residencias o viviendas tuteladas), y (2) tiene efectos positivos en la regeneración urbana, frente al envejecimiento y declive de las barriadas y las limitaciones económicas de la población. Wiesel (2012) sugiere el concepto de envejecimiento positivo como recurso para el renacimiento físico, cultural y espiritual de los barrios urbanos en dos aspectos: primero, las personas mayores disponen de más tiempo libre y pueden participar en actividades, actuando como dinamizadores dentro de la barriada ${ }^{2}$; y segundo, mantienen la memoria colectiva de la barriada como un aspecto fundamental del entorno construido, permitiendo tanto a individuos como a la comunidad conectar con su pasado e identidad (Sandercock, 2003). Sin embargo, actualmente existen tres grandes problemas de este sector de la población: la soledad, la incertidumbre económica de las pensiones y las necesidades especiales de habitabilidad.

Tabla 1. Datos referentes al envejecimiento de las capitales andaluzas y a la oferta de viviendas en alquiler

\begin{tabular}{|c|c|c|c|c|c|c|c|c|c|}
\hline $\begin{array}{c}\text { Capital } \\
\text { andaluza }\end{array}$ & $\begin{array}{l}\text { Hab. } \\
(2017)\end{array}$ & $\begin{array}{c}>65 \\
\text { años en } \\
\text { hab. } \\
(2017)\end{array}$ & $\begin{array}{c}\text { \% de } \\
>65 \\
\text { años } \\
(2017)\end{array}$ & $\begin{array}{c}\text { № de } \\
\text { viv. } \\
\text { prin } \\
\text { (2011) }\end{array}$ & $\begin{array}{c}\text { № de } \\
\text { viv. } \\
\text { alquiler } \\
\text { (2015) }\end{array}$ & $\begin{array}{c}\text { № de } \\
\text { hogares } \\
\text { unipers. } \\
\text { mujer } \\
>65 \\
(2001)\end{array}$ & $\begin{array}{c}\text { № de } \\
\text { hogares } \\
\text { unipers. } \\
\text { hombre } \\
>65 \\
(2001)\end{array}$ & $\begin{array}{l}\text { № total } \\
\text { hogares } \\
\text { unipers. } \\
\quad>65 \\
(2001)\end{array}$ & $\begin{array}{c}\% \text { de } \\
\text { hogares } \\
\text { unipers } \\
>65 \\
(2001)\end{array}$ \\
\hline Sevilla & 689.434 & 130.165 & 18,88 & 268.435 & 42 & 34.472 & 9.536 & 44.008 & 33,81 \\
\hline Málaga & 569.002 & 96.787 & 17,01 & 211.358 & 859 & 27.106 & 9.497 & 36.603 & 37,82 \\
\hline Córdoba & 325.916 & 58.828 & 18,05 & 121.823 & 232 & 7.052 & 1.638 & 8.690 & 14,77 \\
\hline Granada & 232.770 & 48.626 & 20,89 & 96.916 & 97 & 7.234 & 1.424 & 8.658 & 17,81 \\
\hline Almería & 195.389 & 30.442 & 15,58 & 69.178 & 18 & 3.624 & 820 & 4.444 & 14,60 \\
\hline Huelva & 145.115 & 25.221 & 17,38 & 55.255 & 27 & 2.734 & 622 & 3.356 & 13,31 \\
\hline Cádiz & 118.048 & 26.667 & 22,59 & 46.966 & 83 & 3.003 & 704 & 3.707 & 13,90 \\
\hline Jaén & 114.238 & 19.580 & 17,14 & 43.258 & 43 & 2.496 & 526 & 3.022 & 15,43 \\
\hline Andalucía & & & & 913.189 & & 159.213 & 49.065 & 208.278 & 22,81 \\
\hline
\end{tabular}

Fuente: Elaboración propia a partir de datos del Instituto de Estadística y Cartografía de Andalucía, IECA (2019).

El problema de la soledad. Según la Encuesta Continua de Hogares de 2017 (INE, 2018), el $10,62 \%$ de los hogares españoles está habitado por personas mayores que viven en soledad y que supone además el $41,83 \%$ del total de hogares unipersonales. Una tendencia en aumento (en 2017 se produjo un incremento del 1,4\%) y que afecta mayoritariamente a mujeres (1.410.000 hogares) frente a los hombres (550.900 hogares).

En Andalucía, según datos del censo de 2001, más de 200.000 personas viven solas. En un análisis comparativo de las principales ciudades (Tabla 1), el porcentaje más alto se produce en Málaga y Sevilla. Las personas mayores que viven solas lo hacen por el deseo y la satisfacción de continuar en sus viviendas y en su entorno social conocido, dado el alto valor afectivo que los mayores otorgan a la casa, plagada de recuerdos de los que no quieren desprenderse (López Doblas, 2005, pág. 86). El estudio de Costa-Font, Elvira, \& Mascarilla-

\footnotetext{
${ }^{2}$ Por ejemplo, los huertos urbanos del Parque de Miraflores de Sevilla (España), presentada en 1996 en la Conferencia Internacional HABITAT II de las Naciones Unidas son un ejemplo de buenas prácticas ciudadanas. Fueron dinamizadas por la población jubilada procedentes de las migraciones de los años 50 y 60 .
} 


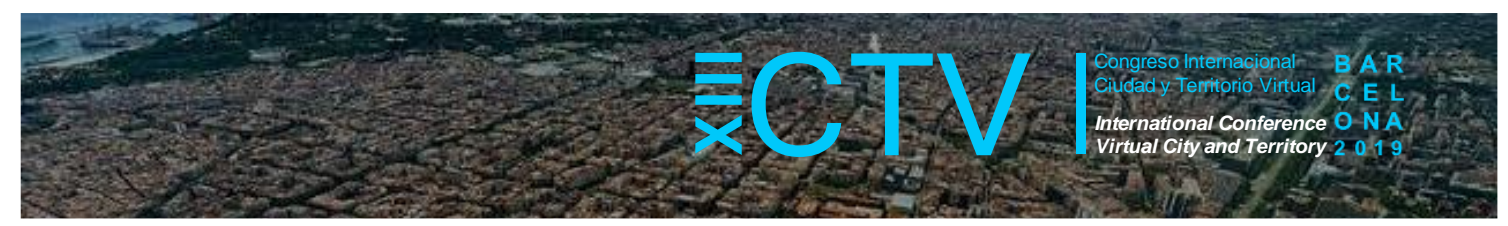

Miró (2009) reveló que el 78\% de las personas mayores prefieren permanecer en sus vivienda, datos que coinciden con estudios internacionales como los de Borgloh \& Westerheide (2012).

Las necesidades habitacionales. Sin embargo, envejecer en el lugar, supone residir en viviendas sobredimensionadas para sus necesidades y, en muchos casos, no adaptadas. En la ciudad esto puede suponer un abandono ya que muchas personas mayores, al quedar viudas (especialmente las mujeres), venden su casa para adquirir otra vivienda de tamaño más reducido y con mejores equipamientos cercana a casa de sus hijos (López Doblas, 2005, pág. 89). Por el contrario, las personas mayores más jóvenes y las personas con movilidad reducida son más proclives a la adaptación de sus viviendas, sin embargo, no todas las personas mayores cuentan con recursos suficientes para poder afrontar una reforma integral.

La incertidumbre económica de las pensiones. El envejecimiento de la población se traduce en una reducción del número de ocupados por pensionista, lo que está obligando a modificar el sistema de pensionas para que sea sostenible financieramente. Ya en 2003, Zuribi Oria (2003) propuso como medidas una reducción del coste mediante el retraso de la edad de jubilación, y el aumento de ingresos mediante la subida de impuestos, cotizaciones o la creación de un fondo de reserva. La reforma del sistema público de pensiones de 2013 ya ha retrasado paulatinamente la edad de jubilación de los 65 a los 67 en el 2027. Aún así se plantea la necesidad de una capitalización complementaria, ya que los escenarios futuros son pesimistas dado el acelerado deterioro financiero del sistema de pensiones. Por ello es preciso buscar modelos que busquen la equidad en términos de distribución del consumo y de paliar la pobreza de la vejez, donde es prioritario aumentar la financiación del sistema sin aumentar los tipos de cotización (Ayuso, Guillén, \& Valero, 2013). De acuerdo con Herce (2016) la creciente esperanza de vida supone un reto de sostenibilidad y suficiencia para las pensiones, públicas o privadas, de capitalización o reparto, que obligará a ir más allá de las importantes reformas adoptadas en los últimos años.

\subsection{La falta de alquileres por efecto de las nuevas economías colaborativas}

Paralelamente, los sectores de población con bajos recursos cuentan con una oferta insuficiente de viviendas en alquiler de larga temporada. En el caso de Málaga capital, según registros del Instituto Municipal de la Vivienda ${ }^{3}$ existían en 2016 aproximadamente 15.000 familias formadas por jóvenes menores de 35 años sin posibilidad del acceso una la vivienda. Una causa importante es el auge de los alquileres vacacionales que ha producido un incremento en los precios de estos alquileres.

La inclusión de la oferta privada colaborativa en el mercado turístico de empresas como AirBNB o Homeaway parece guardar relación directa en el incremento del valor inmobiliario del alquiler de vivienda. El alquiler de larga estancia (destinado principalmente a residentes) sufre una importante competencia por el alquiler de corta estancia (destinado principalmente a visitantes y turistas). El crecimiento exponencial de AirBNB ha alcanzado en 2018 a más de 200.000.000 millones de huéspedes en todo el mundo (Airbnb, 2018). La respuesta de la administración a este fenómeno es el desarrollo de un cuerpo normativo que intenta regularlo -

\footnotetext{
${ }^{3}$ Según entrevista al Gerente el 12/12/2018 (Inédita).
} 


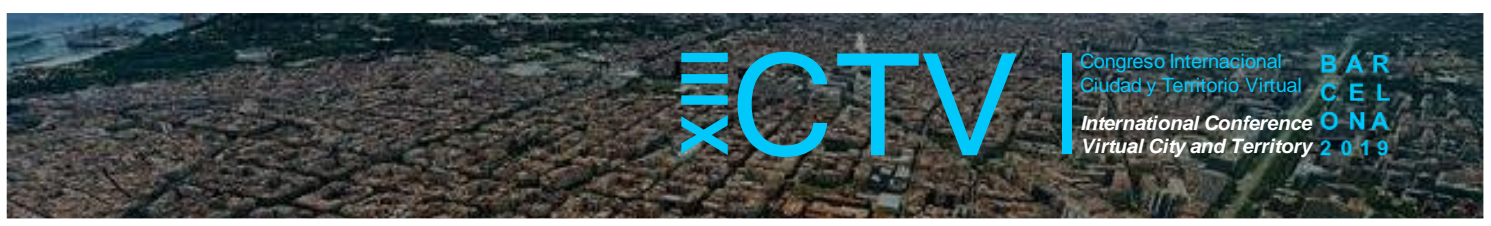

como en el caso de Andalucía ${ }^{4}$ - o prohibirlo. Sin embargo, las administraciones con competencia en materia de vivienda no son capaces de ofrecer nuevos modelos que contrarresten este imparable proceso.

Figura 1. Crecimiento del precio del alquiler de larga temporada en Málaga. En eje de abscisas el año y en ordenadas el precio unitario del alquiler medio $(€ / \mathrm{m} 2)$

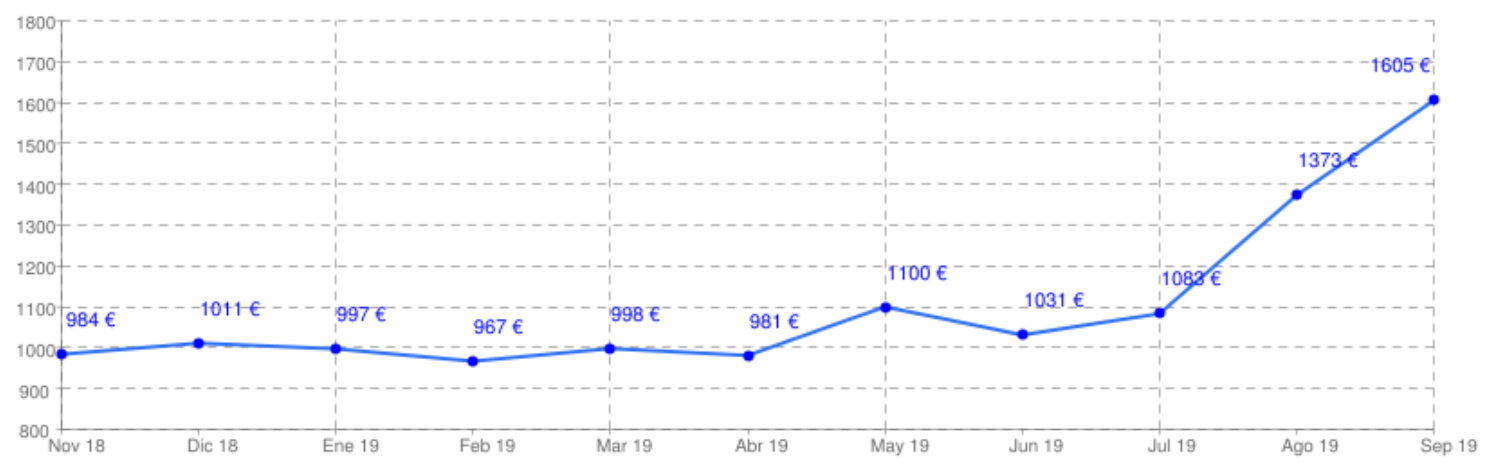

Fuente: Evolución de precio de la vivienda de alquiler (Idealista, 2019)

\subsection{El envejecimiento activo en el lugar y el problema de la co-vivienda para personas mayores}

El envejecimiento activo, entendido como "el proceso de optimización de las oportunidades de salud, participación y seguridad con el fin de mejorar la calidad de vida de las personas que envejecen" (Organización Mundial de la Salud, 2002, pág. 79), ha sido analizado en diversos proyectos de investigación previos como alternativa a los problemas anteriormente detectados.

El problema del envejecimiento, específicamente en los puntos de la falta de financiación y adaptación de las viviendas, ha sido analizado por el proyecto $(R e)$ habitación + $(R e)$ generación + (Re)programación (AOPJA, 2015a), donde se trata la gestión de entornos habitables desde criterios de envejecimiento activo, género y habitabilidad urbana, basado en el diseño de protocolos de intervención en barrios con población mayoritaria de personas mayores, con el fin de garantizar las condiciones de habitabilidad en condiciones de escasa inversión pública. Aunque el proyecto aporta soluciones constructivas de costo reducido, se precisa de un sistema de financiación avalado por la administración, que sólo garantiza el pago de una inversión que finalmente deben de asumir los escasos recursos de las personas mayores.

Frente a la alternativa a vivir solo, el envejecimiento activo evita el desplazamiento de las residencias, la rotación periódica entre familiares o la búsqueda de parejas, destacamos el proyecto de investigación Nuevas propuestas habitacionales para promover el envejecimiento activo (AOPJA, 2015b), que analizó las posibilidades de la creación de co-vivienda (cohousing) como modelo habitacional para la promoción del envejecimiento activo. En palabras de Choi (2004) una co-vivienda es un modelo de vivienda en el cual algunas personas comparten funciones importantes de la vida diaria, espacios de relación y es también una forma de "cooperativa" al ser el modelo más utilizado para su configuración. Las políticas estatales de vivienda a través del reciente Plan estatal de la vivienda (2018-21) ya incluyen el modelo de covivienda como una alternativa habitacional para el grupo de "especial protección de los

\footnotetext{
${ }^{4}$ Decreto 28/2016, de 2 de febrero, de las viviendas con fines turísticos y de modificación del Decreto 194/2010, de 20 de abril, de establecimientos de apartamentos turísticos. BOJA no28 (11/02/2016).
} 


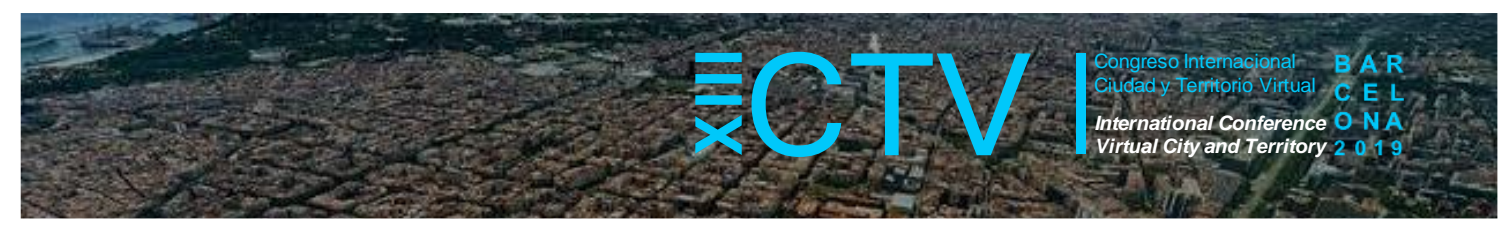

mayores de 65 años, mediante el fomento de conjuntos residenciales de viviendas con instalaciones y servicios comunes adaptados, en régimen de alquiler, de cesión en uso con renta o precio limitado" (PEV, 2019, pág. 28900). Específicamente el "Programa de fomento de viviendas para personas mayores y personas con discapacidad" facilita -además de la tradicional subvención de la accesibilidad de los edificios existentes-, la creación de coviviendas para personas mayores definidas como "las promociones de viviendas de nueva construcción o de viviendas procedentes de la rehabilitación de edificios que se vayan a ceder en uso o destinar al arrendamiento, en ambos supuestos, por un plazo de al menos cuarenta años" (PEV, 2019, pág. 28905). El programa de co-vivienda debe "incluir instalaciones y servicios mínimos que como mínimo: asistencia social, atención médica básica veinticuatro horas disponible y cercana, limpieza y mantenimiento, dispositivos y sistemas de seguridad, restauración, actividades sociales, deportivas, de ocio y culturales, así como terapias preventivas y de rehabilitación" (PEV, 2019, pág. 28905).

La co-vivienda es una opción muy positiva de proyecto de convivencia, sin embargo, existen tres problemas en la implantación de este modelo como alternativa para la soledad de las personas mayores:

a) No todas las personas mayores desean este modelo, para se relaciona más con los jóvenes mayores de 50-65 (Choi, 2004; Brenton, 2008) al contar con un mayor interés en esta forma de organización.

b) El modelo está orientado a la construcción de unas nuevas promociones que respondan a las necesidades del programa de convivencia que diseñen sus miembros. Aunque existen coviviendas basadas en la rehabilitación, estas se producen en un porcentaje todavía muy reducido. Las dificultades de encontrar suelo o un edificio completo obligan a que la futura comunidad se desplace a la periferia de las ciudades, atraídos también por mejores condiciones ambientales (un ejemplo de ello es la cooperativa Los Milagros en Málaga).

c) Por tanto, al ser la implementación en las barriadas obsoletas un proceso complejo, es muy posible que se produzca un riesgo de descapitalización social de sus residentes, al desplazarse sus activos más dinámicos hacia la periferia.

\subsection{Las cooperativas vecinales de personas mayores como modelo teórico alternativo}

La cooperativa es uno de los mejores exponentes de la economía social, dado su carácter no lucrativo y compromiso social derivado de su modo de gobernanza. Es considerado por la Unión Europa como un agente de salida estructural de la crisis económica por su capacidad de regular los numerosos desequilibrios del mercado laboral (desempleo, inestabilidad laboral, exclusión social, etc.) y su capacidad de movilizar el potencial económico endógeno, construyendo colectivamente nuevas sinergias para la revitalización de las zonas locales.

Efectivamente, la Estrategia Europa 2020 establece tres prioridades básicas: crecimiento inteligente, crecimiento sostenible y crecimiento integrador. Las cooperativas han demostrado que contribuyen al desarrollo de una economía basada en el conocimiento y la innovación (crecimiento inteligente); cuentan con sistemas de valores que materializan la solidaridad con el entorno, internalizando los costes sociales y generando externalidades positivas (crecimiento 


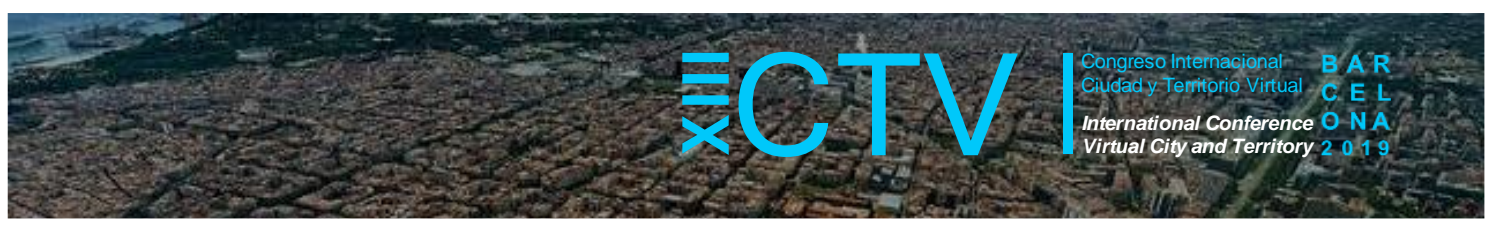

sostenible); y en el ámbito de la lucha contra la pobreza y la exclusión social, el auge de las empresas sociales ha sido considerable (crecimiento integrador). Además la capacidad de las cooperativas para resolver las nuevas necesidades sociales ha revitalizado su importancia (Monzón \& Chaves, 2012, pág. 95).

Las cooperativas vecinales son un modelo de gestión de regeneración urbana basada en la creación de una cooperativa de consumo y trabajo para la prestación de servicios de habitabilidad para una barriada o un conjunto de ellas, donde participan todos los residentes (propietarios o no) así como los comercios locales que allí residen (Rosa-Jiménez, MárquezBallesteros, \& Navas-Carrillo, 2017). En este ámbito, se detectó el potencial de la cooperativa de personas mayores para atender a dos graves problemas actuales: el incremento constante de personas mayores que viven solas y la escasez de vivienda para alquilar. Dos aspectos que la creación de una cooperativa vecinal permite resolver de forma conjunta, al relacionar ambos procesos.

Figura 2. Modelo teórico de una cooperativa vecinal de personas mayores

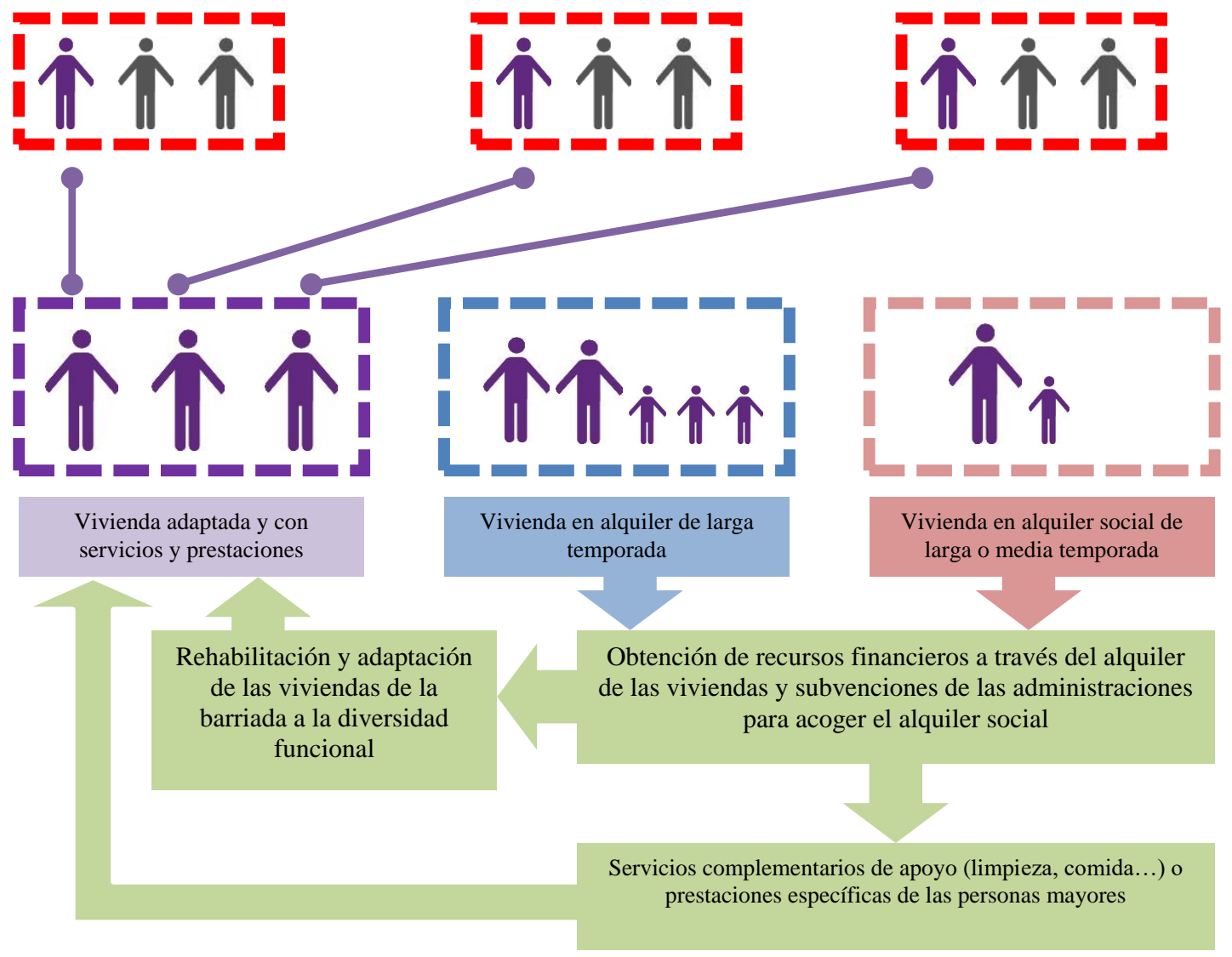

Fuente: elaboración propia.

A tenor de los problemas planteados, la hipótesis de partida es que la creación de una cooperativa vecinal de personas mayores en una barriada es un modelo viable. Resuelve el problema de la soledad mediante el agrupamiento de cooperativistas en viviendas adaptadas, liberando el resto de viviendas que pasarían a formar parte de la oferta de viviendas de alquiler de larga temporadas que sería gestionada por la propia cooperativa. De esta forma, las 


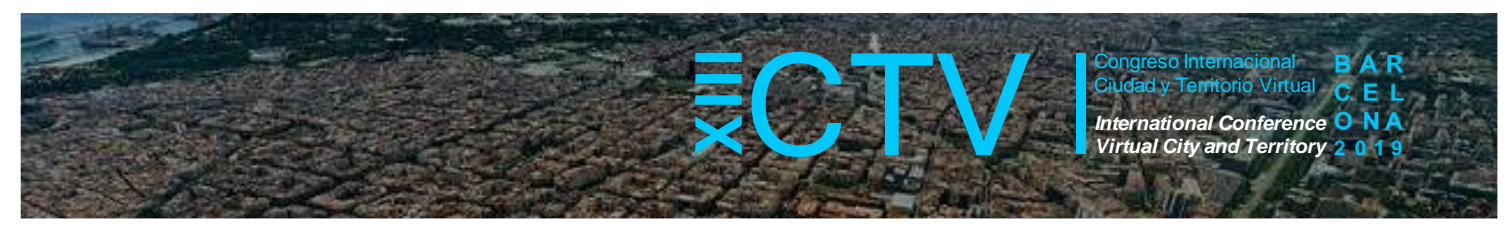

personas mayores podrían adaptar aquellas viviendas que estuvieran mejor localizadas para sus necesidades, mientras que el alquiler de las viviendas restantes permitirían la obtención de recursos económicos que permita la capitalización de los recursos económicos de las personas mayores, mediante la economía social, como una forma de obtener ingresos, pero también como una herramienta que pueden utilizar las administraciones para intervenir el mercado de vivienda de alquiler, especialmente para aquellas familias con peligro de exclusión social.

El modelo teórico de cooperativa vecinal de personas mayores está reflejado en la Figura 2. Se basa en dos procesos: el primero de agrupación de socios en una sola vivienda que se rehabilita y adapta a las personas mayores; y el segundo, el de obtención de ingresos a partir de la oferta de alquiler de larga temporada de las viviendas desocupadas. Con los ingresos obtenidos se sufragan los gastos de rehabilitación y además permite la contratación de servicios complementarios, como personal de apoyo para las personas mayores.

\section{Metodología}

Para validar el modelo teórico se hace un estudio económico de las ventajas de su implantación en una barriada. Se toma como caso de estudio barriadas de la ciudad de Málaga como ejemplo de ciudad andaluza con niveles elevados de personas mayores (ver Tabla 1). Se obtienen datos de la característica de la barriada y del Instituto de Estadísticas y Cartografía de Andalucía (IECA, 2019); así como los datos del mercado de alquiler turísticos a partir de las plataformas electrónicas que las ofrecen Fotocasa (2019). Para el estudio económico se hace un estudio de ingresos y gastos. En los costes de reforma de las viviendas se aplica la estimación porcentual por capítulos para proyectos parciales y/o reformados según los costes de referencia del Colegio de Arquitectos de Málaga (COAM, 2019). Según estos, se estima que la adaptación de locales a uso específico es un $50 \%$ sobre el coste de referencia para el uso proyectado para calidades básicas equivalentes a viviendas de promoción oficial. El proyecto contempla que el servicio de mantenimiento asociado a cada inversión sea ofertado dentro del contrato de adjudicación de las acciones de mejora o la prestación del servicio. A diferencia del caso de las instalaciones, las soluciones constructivas para el interior de las viviendas se les exigen menos acciones de mantenimiento, lo que permite estimar su coste en un $10 \%$ del presupuesto de ejecución material.

\section{Resultados}

\subsection{Implantación del modelo en el caso de estudio}

Estudiamos la implantación en las barriadas de Sixto y Cortijo Vallejo en Málaga (España). Son barriadas que se localizan en la zona occidental de la ciudad, en el distrito de Carretera de Cádiz: una gran extensión de residencial construida principalmente durante los años 1960 y 1970, para absorber las migraciones del campo-ciudad. Desarrollado prácticamente en su totalidad con viviendas en bloques aislados de gran altura y con subvenciones oficiales. La barriada de Sixto se construyó a principios de 1960, en bloques de cinco plantas sin ascensor; mientras que la barriada de Cortijo Vallejo cuenta con 10 plantas y ascensor. Suman aproximadamente un total de 2.000 habitantes y en ambas barridas existen 114 viviendas en las que viven solas personas mayores de 65 años. 


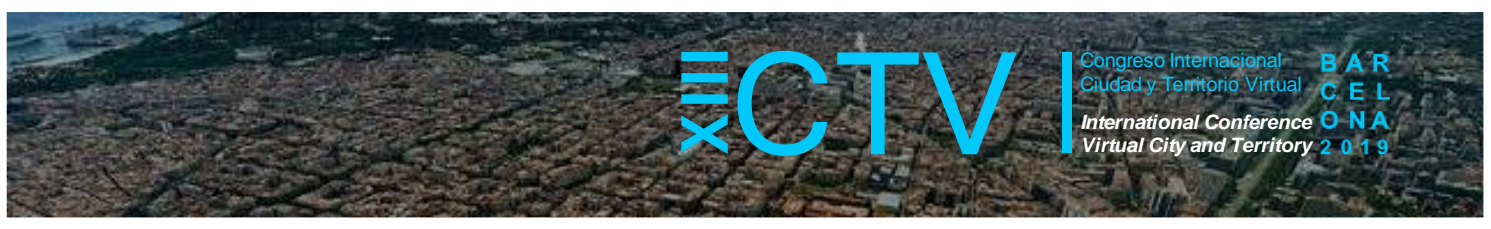

El grado de intervención necesaria determina que prioritariamente se deben modificar baños, cocinas y zonas de paso. Además, se requerirá la modificación de la compartimentación de dormitorios para adecuar sus dimensiones por lo que se estima para los cálculos que la totalidad de la superficie de la vivienda se verá afectada por la intervención. Las viviendas de la barriada de Sixto son viviendas mínimas que se pueden reformar para un programa de dos personas, con 1 baño adaptado, 2 dormitorios de similares dimensiones y comedor-cocina. Mientras que las viviendas de Cortijo Vallejo por lo general, son tipologías residenciales de 4 dormitorios, adecuadas para adaptar el programa para albergar 3 personas, mediante la redistribución interior para acoger 1 baño adaptado, 3 dormitorios de similares dimensiones y comedor-cocina.

\subsection{Ingresos por gestión viviendas liberadas}

De las 114 viviendas de personas que viven solas, y de acuerdo con las características tipológicas de ambas barriadas, es posible agrupar socios en 46 viviendas adaptadas lo que permite poner 68 vivienda en el mercado de alquiler (Tabla 2). El precio medio del alquiler para las viviendas de Sixto y Cortijo Vallejo se han fijado respectivamente en $400 €$ y $450 €$ (IVA excluido), unos precios muy por debajo de los precios de mercado existentes en la zona que rondan los $9 € / \mathrm{m} 2$ (Figura 3).

Figura 3. Precio medio de alquiler en la zona de estudio de Málaga (España)

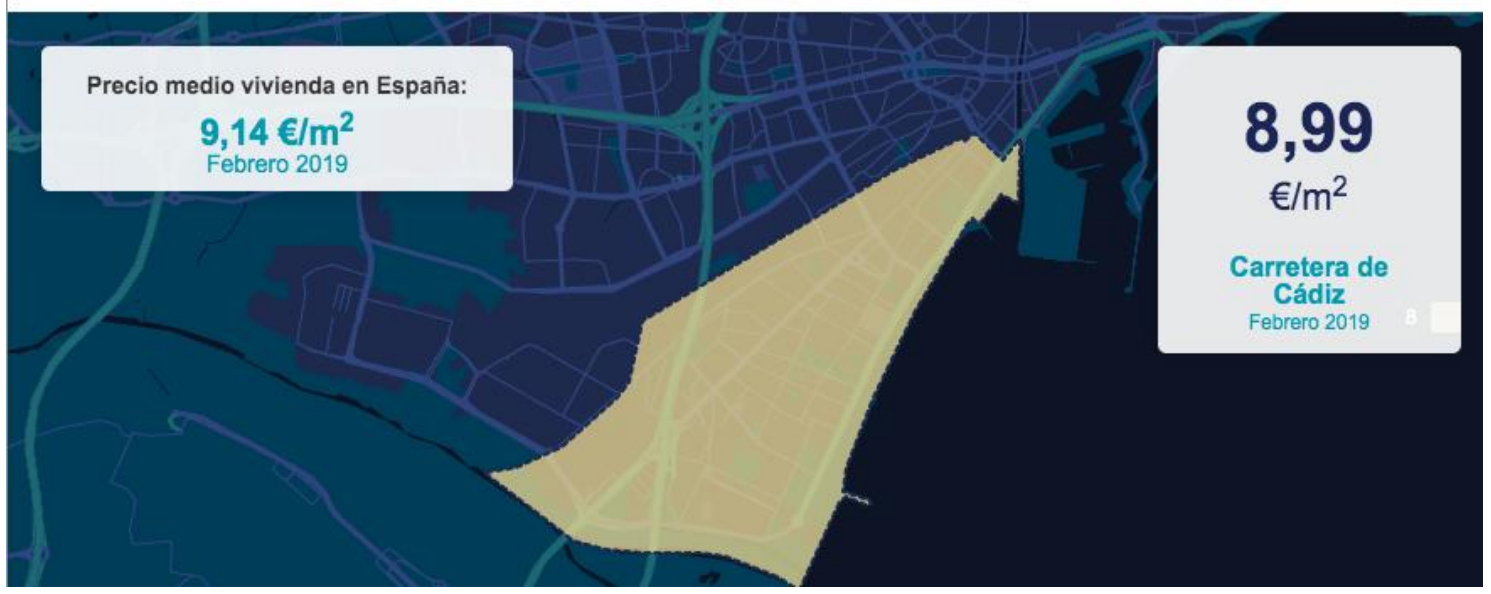

Fuente: Fotocasa (2019)

Tabla 2. Distribución de las viviendas de las personas mayores en la constitución de la cooperativa vecinal de personas mayores

\begin{tabular}{lllllll}
\hline & \multicolumn{2}{l}{$\begin{array}{l}\text { Agrupación de personas mayores } \\
\text { para evitar la soledad }\end{array}$} & \multicolumn{4}{l}{$\begin{array}{l}\text { Oferta de viviendas para alquiler } \\
\text { de larga estancia }\end{array}$} \\
\hline Barriada & $\begin{array}{l}\text { № viviendas } \\
\text { adaptadas }\end{array}$ & $\begin{array}{l}\text { № de socios } \\
\text { por vivienda } \\
\text { adaptada }\end{array}$ & $\begin{array}{l}\text { Total de socios } \\
\text { agrupados }\end{array}$ & $\begin{array}{l}\text { № viviendas } \\
\text { liberadas } \\
\text { para alquiler }\end{array}$ & $\begin{array}{l}\text { Alquiler } \\
\text { mensual (€) }\end{array}$ & $\begin{array}{l}\text { Ingreso } \\
\text { anual (€) }\end{array}$ \\
\hline Sixto & 24 & 2 & 48 & 24 & 400 & 115.200 \\
\hline Cortijo Vallejo & 22 & 3 & 66 & 44 & 450 & 237.600 \\
\hline TOTAL & $\mathbf{4 6}$ & - & $\mathbf{1 1 4}$ & $\mathbf{6 8}$ & & $\mathbf{3 5 2 . 8 0 0}$ \\
\hline
\end{tabular}

Fuente: elaboración propia. 


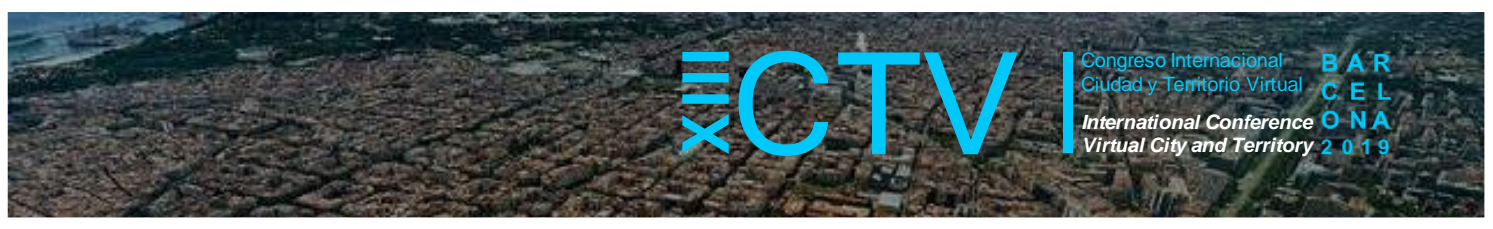

\subsection{Costes por adaptación de las viviendas agrupadas}

La constitución de una cooperativa permite aplicar una reducción del coste de construcción por economía de escala. La superficie a adaptar de las viviendas queda reflejada en la Tabla 3. La importante superficie total para adaptar a los criterios del Código Técnico de la Edificación española y de las normas de accesibilidad universal, permite obtener más ventajas que si éstas se realizaran de forma independiente por cada uno de los propietarios, tal y como queda reflejado en la Tabla 4. Dichos ahorros se resumen en tres:

Tabla 3. Superficie construida de las viviendas adaptadas

Fuente: elaboración propia.

\begin{tabular}{llrr}
\hline Barriada & № viv. & Sup. $(\mathbf{m} 2)$ & Total Sup. Cons $\mathbf{( m 2 )}$ \\
\hline Sixto & 18 & 65,00 & 1170,00 \\
\hline & 6 & 55,00 & 330,00 \\
\hline Cortijo Vallejo & 6 & 85,00 & 510,00 \\
\hline & 10 & 109,00 & 1090,00 \\
\hline & 6 & 75,00 & 450,00 \\
\hline TOTAL & $\mathbf{4 6}$ & - & $\mathbf{3 5 5 0 , 0 0}$ \\
\hline
\end{tabular}

a. Ahorro por eficiencia técnica. El número de viviendas a adaptar es el mismo para ambas hipótesis por lo que no se estima una reducción en el total de la superficie sobre la que actuar. Sin embargo, sí debe tenerse en cuenta que el porcentaje de honorarios técnicos puede descender del $18 \%$ al $11 \%$ sobre el presupuesto de ejecución material, al considerar una intervención conjunta y poder aplicar un factor de repetición de las soluciones adoptadas.

b.

c. Ahorro por eficiencia económica. Se ha estimado una mejora económica del $25 \%$ por oferta competitiva de la empresa que licite la obra y el mantenimiento de las viviendas, de forma similar a los procedimientos ordinarios de contratación pública.

d.

c. Reducción en las tasas e impuestos municipales. El modelo asume la bonificación de las tasas e impuestos municipales al 100\% con base en el artículo 6 de la Ley de Ordenación Urbana de Andalucía, por el cual los ayuntamientos podrán aplicar bonificaciones sobre las tasas por expedición de licencias de obras para la conservación o rehabilitación de la edificación.

Tabla 4. Costes de adaptación de las viviendas para cada una de las hipótesis de estudio

\begin{tabular}{llrrrrr}
\hline Hipótesis & Agente & Base $(\boldsymbol{\epsilon})$ & Desc. $(\%)$ & Base reducida $(\boldsymbol{\epsilon})$ & IVA $(\boldsymbol{\epsilon})$ & \multicolumn{1}{c}{ Total $(\boldsymbol{\epsilon})$} \\
\hline Sin & Contratista & 1.551 .000 & - & - & 325.000 & 1.876 .000 \\
\hline Cooperativa & Proyectista & 216.000 & - & - & 45.000 & 261.000 \\
\hline & Ayuntamiento & 78.100 & - & - & - & 78.000 \\
\hline & TOTAL & $\mathbf{1 . 8 4 5 . 0 0 0}$ & - & - & $\mathbf{3 7 0 . 0 0 0}$ & $\mathbf{2 . 2 1 5 . 0 0 0}$ \\
\hline Con & Contratista & 1.600 .000 & 25 & 1.200 .000 & - & 1.200 .000 \\
\hline Cooperativa & Proyectista & 160.000 & 18 & 132.000 & - & 132.000 \\
\hline & Ayuntamiento & 78.000 & 100 & - & - \\
\hline & TOTAL & $\mathbf{1 . 8 3 8 . 0 0 0}$ & & $\mathbf{1 . 3 3 2 . 0 0 0}$ & $\mathbf{1 . 3 3 2 . 0 0 0}$ \\
\hline
\end{tabular}

Fuente: elaboración propia.

Según se observa en la Tabla 4 , el ahorro estimado es de $883.000 €$, ya que además la Cooperativa Vecinal no repercute el IVA, esto supone casi un $40 \%$ de ahorro. El gasto de la 


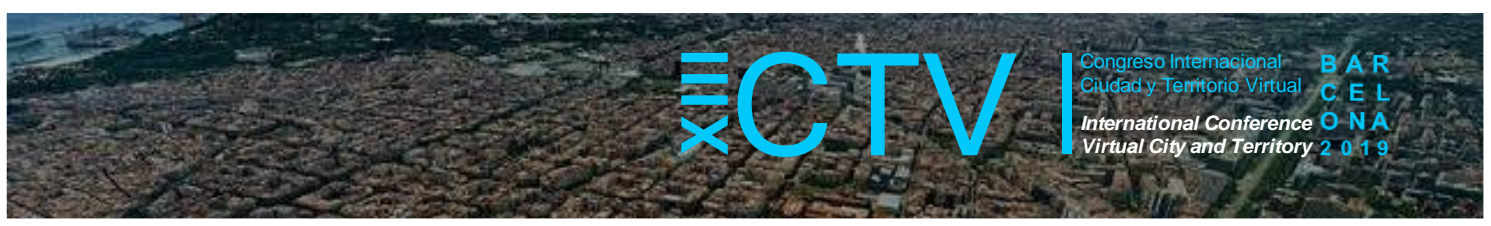

rehabilitación para un plazo de amortización de 10 años y, con un interés muy reducido o subvencionado se puede establecer en $136.000 € /$ año.

\subsection{Viabilidad económica}

Como se observa en el resumen de ingresos y gastos de la Tabla 5. Los ingresos obtenidos por el alquiler de las viviendas liberadas permiten, por un lado, financiar los gastos de rehabilitación y, por otro lado, el abono de una serie de servicios: la contratación de 11 auxiliares de clínica -lo que supone una ratio aproximada de 1 auxiliar por cada 10 socios-, además de contar con recursos económicos para la propia gestión de la cooperativa.

Tabla 5. Viabilidad económica de la cooperativa para una amortización a 10 años

\begin{tabular}{lrr}
\hline Concepto & Ingresos (€) & Gastos (€) \\
\hline Alquiler de 68 viviendas & 352.800 & \\
\hline $\begin{array}{l}\text { Rehabilitación y adaptación de 46 viviendas para agrupar las personas mayores en } \\
\text { unidades de } 2 \text { o } 3 \text { personas por vivienda (amortización a 10 años) }\end{array}$ & 136.000 \\
\hline Gestión de la cooperativa & 51.800 \\
\hline Sueldo bruto anual de 11 auxiliares (15.000 €/año) & 165.000 \\
\hline TOTAL & $\mathbf{3 5 2 . 8 0 0}$ & $\mathbf{3 5 2 . 8 0 0}$ \\
\hline
\end{tabular}

Fuente: elaboración propia.

\section{Conclusiones}

El modelo de cooperativa vecinal de personas mayores permite resolver los siguientes problemas del envejecimiento en el lugar:

(a) Evita la soledad de las personas mayores de una barriada, agrupando en viviendas compartidas de dos o tres personas, bien en la propia vivienda o en viviendas cercanas, permitiendo con ello el envejecimiento en el lugar. A diferencia del modelo de co-vivienda, las personas no precisan abandonar el barrio.

(b) Adapta sus necesidades habitacionales, ya que las viviendas son rehabilitadas y reformadas con nuevos programas habitacionales -más cercanos a las personas mayores-, e incorpora además prestaciones asistenciales de apoyo en la limpieza o comidas.

(c) Supera la incertidumbre económica de las pensiones, en el sentido que el modelo es autosuficiente económicamente, por lo que los socios no deben de hacer inversiones económicas. Cuestión que si ocurriría si deben de abordar la rehabilitación de su vivienda de forma individual.

Respecto al mercado de vivienda el beneficio es doble: por un lado, incrementa la rehabilitación del parque de viviendas para personas mayores y, por otro, incrementa el mercado de alquiler a precio tasado. El fomento de este modelo por parte de las administraciones públicas, puede ser una fórmula a tener en cuenta, previo al desarrollo de políticas de construcción de vivienda pública para el alquiler social. Si extrapolamos los resultados obtenidos, aproximadamente el $50 \%$ de las personas mayores que viven solas pueden ofrecer su vivienda al alquiler de larga temporada que, en el caso de Málaga, puede representar una oferta de 18.000 viviendas de alquiler, y el de Andalucía hasta un total de 100.000. 


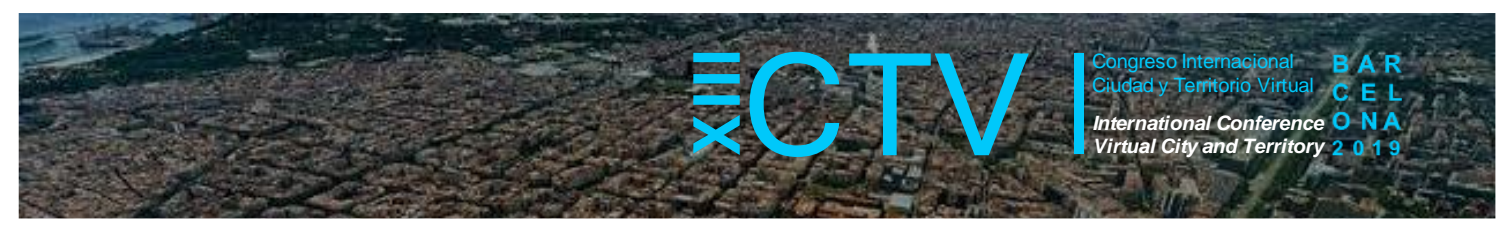

El modelo cuenta con limitaciones. Por un lado, es un modelo para personas mayores no dependientes y con autonomía, pero que deben de estar preparadas para asumir que comparten su vivienda con otras personas. Por otro lado, aunque las personas mayores siguen viviendo en el barrio, no todas lo hacen en su vivienda, por lo que puede haber resistencia a abandonar la misma. Además, se produce una discriminación: las personas que rehabilitan sus viviendas se pueden sentir más beneficiadas que aquellas que ofrecen su vivienda en alquiler. Para futuras investigaciones, sería de especial interés, resolver estas cuestiones además de determinar el tamaño óptimo de la cooperativa en función de la capacidad de obtener recursos a partir del mercado de alquiler, la adaptación física de los espacios públicos y equipamientos urbanos de las barriadas para permitir un envejecimiento en el lugar; así como el desarrollo de redes sociales tecnológicas que faciliten la relación de los cooperativistas.

Agradecimientos: Este trabajo está financiado por el proyecto de investigación "Gestión cooperativa en la revitalización de barriadas y en la oferta de servicios de habitabilidad. Formulación y aplicabilidad", concedido en el marco del I Plan Propio de Investigación y Transferencia de la Universidad de Málaga (España).

Contribuciones de los autores: El primer autor ha contribuido a la concepción y diseño del estudio, adquisición, análisis e interpretación de los datos, así como a la redacción del texto; la segunda autora ha llevado a cabo una revisión crítica del artículo.

Conflicto de Intereses: Los autores declaran que no hay conflicto de intereses.

\section{Bibliografía}

Abellán García, A., Ayala García, A., \& Pujol Rodríguez, R. (2017). Un perfil de las personas mayores en España, 2017. Indicadores estadísticos básicos. Informes Envejecimiento en red (Vol.15).

Agencia de Obra Pública de la Junta de Andalucía. AOPJA. (2015a). (Re)habitación +

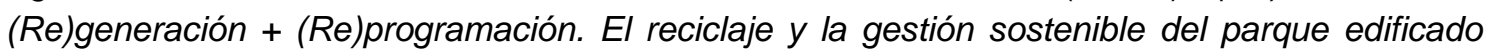
andaluz. Gestión de entornos habitables desde criterios de envejecimiento activo, género y habitabilidad urbana (GG/3001IDIJ) Año 2011-15. [Archivo de datos y documentos]. Recuperado de http://www.aopandalucia.es/innovacion/principal.asp?alias=(RE) Programa

Agencia de Obra Pública de la Junta de Andalucía. AOPJA. (2015b). Nuevas propuestas habitacionales para promover el envejecimiento activo. [Archivo de datos y documentos]. Recuperado de http://www.aopandalucia.es/innovacion/principal.asp?alias=cohousing taller\&t=0\&zona=areas

Airbnb (2018). www.airbnb.es/ Accedido el 16 de enero de 2018.

Ayuso, M., Guillén, M., \& Valero, D. (2013). Sostenibilidad de las pensiones en España desde la perspectiva de la equidad y la eficiencia. Presupuesto y Gato Público, 71, 193-204.

Borgloh, S., \& Westerheide, P. (2012). The Impact of Mutual Support Based Housing Projects on the Costs of Care. Housing Studies, 27(5), 620-642. DOI https://doi.org/10.1080/02673037.2012.697554 


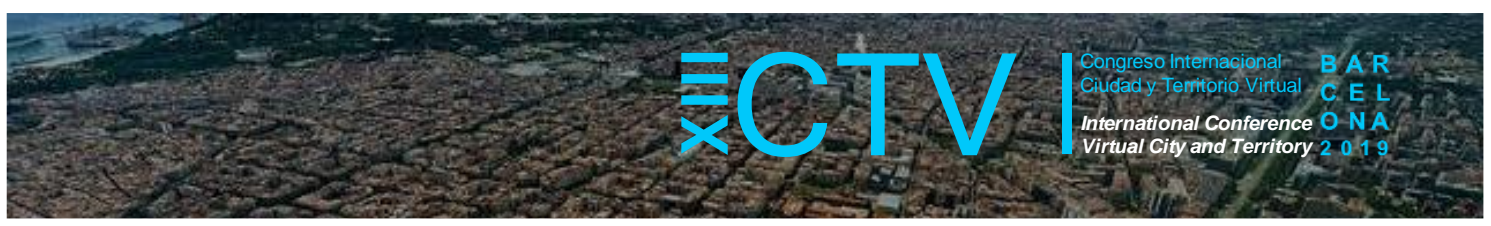

Choi, J. S. (2004). Evaluation of Community Planning and Life of Senior Cohousing Projects in Northern European Countries. European Planning Studies, 12(8), 1189-1216. DOI https://doi.org/10.1080/0965431042000289296

COAM. (2019). Valores medios estimativos de la Construcción 2019. Recuperado 3, 2019, de http://coamalaga.es/wp-content/uploads/2019/01/VALORES-MEDIOS-ESTIMATIVOS-DE-LACONSTRUCCIÓN-2019.pdf

Costa-Font, J., Elvira, D., \& Mascarilla-Miró, O. (2009). "Ageing in place"? Exploring elderly people's housing preferences in Spain. Urban Studies, 46(2), 295-316. DOI https://doi.org/10.1177/0042098008099356

Decreto 28/2016, de 9 de diciembre, de las viviendas con fines turísticos y de modificación del Decreto 194/2010, de 20 de abril, de establecimientos de apartamentos turísticos. Boletín Oficial de la Junta de Andalucía, núm. 28, de 11 de febrero de 2016. Recuperado de https://www.juntadeandalucia.es/boja/2016/28/6

Fernández Muñoz, J. N. (2015). Informe 2014. Las personas Mayores en España. Datos estadísticos estatales y por Comunidades Autonómas. Madrid.

Fotocasa.

(2019).

Recuperado

de

https://www.fotocasa.es/indice/\#/filter/eyJpZCI6MTUwNSwidHIwZSI6NCwidHJhbnNhY3Rpb24i OiJyZW50In0. Accedido el 16 de junio de 2019.

Herce, J. A. (2016). El impacto del envejecimiento de la población en España. Cuadernos de Información Económica, 251, 39-48. Recuperado de http://www.ine.es/daco/daco42/sociales/infosoc envej.pdf

Hui, E. C. M., Wong, F. K. W., Chung, K. W., \& Lau, K. Y. (2014). Housing affordability, preferences and expectations of elderly with government intervention. Habitat International, 43, 11-21. https://doi.org/10.1016/j.habitatint.2014.01.010

Idealista. (2019). www.idealista.com. Accedido el 15 de mayo 2019.

IECA. (2019). Instituto de Estadística y Cartografía de Andalucía. https://www.juntadeandalucia.es/institutodeestadisticaycartografia/temas/index-est.htm.

Accedido el 5 de mayo 2019.

INE. (2018, April 12). Encuesta Continua de Hogares. Año 2017. Notas de Prensa Del INE, pp. 2-11. Madrid. Recuperado de http://www.ine.es/prensa/np965.pdf

López Doblas, J. (2005). Personas mayores viviendo solas. La autonomía como valor en alza. Imserso. Madrid: IMSERSO.

Monzón, J. L., \& Chaves, R. (2012). La Economía Social En La Unión Europea. Informe.

Organización Mundial de la Salud. (2002). Envejecimiento activo: un marco político. Revista Española de Geriatría y Gerontología, 37(S2), 74-105. Recuperado de http://envejecimiento.csic.es/documentos/documentos/oms-envejecimiento-01.pdf 


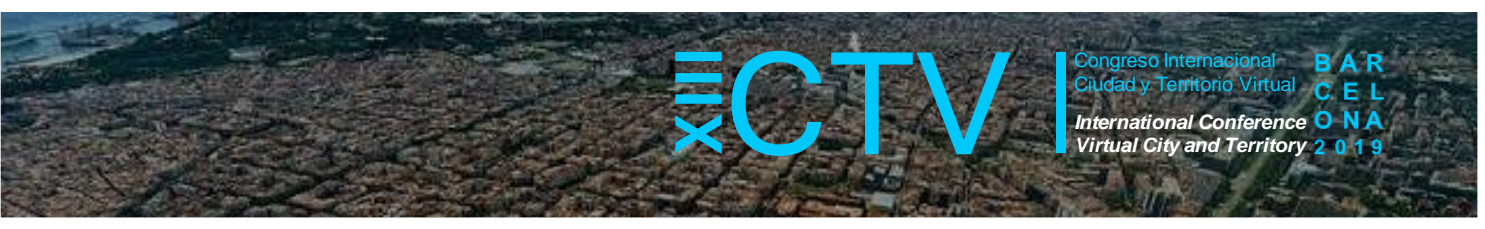

PEV (2018). Real Decreto 106/2018, de 9 de marzo, por el que se regula el Plan Estatal de Vivienda 2018-2021. Boletín Oficial del Estado, núm. 61, de 10 de marzo de 2018, pp. 2886828916. Recuperado de https://www.boe.es/eli/es/rd/2018/03/09/106

Rosa-Jiménez, C., Márquez-Ballesteros, M., \& Navas-Carrillo, D. (2017). Hacia un nuevo modelo de gestión y autofinanciación de la regeneración de barriadas obsoletas. Ciudades, 20, 45-70. DOI https://doi.org/10.24197/ciudades.20.2017.26

Sandercock, L. (2003). Towards Cosmopolis: Utopia as construction site. In Readings in Planning Theory (pp. 401-407). Melbourne: Blackwell.

Wiesel, I. (2012). Can Ageing Improve Neighbourhoods? Revisiting Neighbourhood Life-Cycle Theory. Housing Theory Society, 29(2), 145-156. DOI https://doi.org/10.1080/14036096.2011.641258

Zuribi Oria, I. (2003). El futuro del sistema de pensiones en España. Madrid1a: Instituto de Estudios Fiscales. 